importance of living in harmony with nature, acting on the instant instincts, and realizing the limits that vary from person to person.

Thuppil Venkatesh, MD

Bangalore, India

\section{Endogenous Antioxidants and Three Paradoxes of Hypoxic Preconditioning}

Severe acute hypobaric hypoxia increases the expression of 4 endogenous antioxidant proteins ( $\mathrm{Cu}, \mathrm{Zn}-\mathrm{SOD}, \mathrm{Mn}-\mathrm{SOD}$, thioredoxin-1 [Trx-1], and thioredoxin-2 [Trx-2]) in rat hippocampus. This increase is a protective reaction, but it is not enough to prevent massive delayed neuronal death. A preconditioning by 3 episodes of mild hypoxia (3PC) significantly enhances and, in some cases, accelerates the increase of antioxidant expression after subsequent severe hypoxia. That prevents neuronal death and ameliorates functional disorders induced by severe hypoxia. It has been assumed that the effect of 3PC is associated with protective increase of "background" antioxidant levels even before severe hypoxia. The first paradox, however, is that $3 \mathrm{PC}$ itself (ie, without or before subsequent severe hypoxia) does not increase the expression of Trx-1, Trx-2, or Cu,Zn-SOD in any hippocampal area 24 hours after last session (ie, at the starting point of severe hypoxia). Moreover, in many cases, 3PC itself significantly reduces the hippocampal expression of these antioxidants. We have hypothesized that the neuroprotective effect of preconditioning bears on the reduced "background" levels of antioxidants, which facilitates reactive oxygen species-mediated signal transduction.

The second paradox is that preconditioning by a single episode of mild hypoxia (1PC), in contrast to 3PC, does not have a neuroprotective effect against subsequent severe hypoxia. Nonetheless, the effects of $1 \mathrm{PC}$ itself on the expression of antioxidants are similar to the effects of 3PC. Thus, the neuroprotective effects of preconditioning in these cases appear not to be determined by any specific baseline level of antioxidant expression at the starting timepoint of subsequent severe hypoxia but, rather, are caused by dynamic changes of the expression, which switch the neuronal cells into a functional state of readiness for rapid and intense expression of antioxidants in response to severe hypoxia.

The third paradox is that the 6-trial mild hypoxia (6PC) itself significantly amplifies the expression of Trx-1 and both SODs. In case of subsequent severe hypoxia, 6PC has a neuroprotective effect, albeit less efficient than 3PC. However, the mechanism of neuroprotection essentially differs. In 6PC, the neuroprotective effect is apparently caused by accumulation of a defensive pool of antioxidants by the starting point of severe hypoxia.

Stroev Sergei Alexandrovich, $\mathrm{PhD}$ Tampere, Finland and St. Petersburg, Russia
Tyulkova Ekaterina Iosifovna, $\mathrm{PhD}$

Glushchenko Tatiana Sergeevna, $\mathrm{PhD}$ Samoilov Mikhail Olegovich, MD, PhD

St. Petersburg, Russia

\section{Use of Intermittent Hypoxia Training (IHT) in Cardiology: Principles and Practices}

To the present, intermittent hypoxia training (IHT) has been used extensively for altitude preacclimatization, in sport practice, and for treating a variety of clinical disorders, including coronary artery disease and arterial hypertension, as well as a tool to prepare patients for coronary interventions. Many investigations are primarily focused on the detrimental effects of intermittent hypoxia associated with obstructive sleep apnea (OSA). The following questions arise: Why do OSA and IHT produce such disparate effects on the cardiovascular system? What are the key mechanisms determining the adaptive versus maladaptive nature of different hypoxic paradigms?

An impressive amount of scientific information has been gathered, from the integrative systems level to the molecular and genomic level, but there is no exact evidence of the precise mechanism for switching adaptive or maladaptive responses to hypoxic impact. Nevertheless, basic investigations led to the introduction of various IHT methods in clinical practice and to the development of different medical equipment, named hypoxicators, for their implementation. In particular, clinical data indicate that, in patients with coronary artery disease, IHT reduces daily myocardial ischemia, blood viscosity and platelet aggregation, increases hypoxic tolerance, improves endothelial function, normalizes systemic blood pressure, and so forth.

Recently, a new mode of adaptive training was explored that combines periods of hypoxia and hyperoxia. A novel principle of short-term periodic adaptive training by varying the oxygen level from hypoxia to hyperoxia is substantiated both theoretically and experimentally. We can envisage a bright future for individualized IHT, which may play a significant role in the quickly developing field of personalized preventive medicine against cardiovascular diseases.

Tatiana Serebrovska, $\mathrm{PhD}$ Valery B. Shatilo, $\mathrm{PhD}$ Kiev, Ukraine

\section{Surviving Birth at Any Altitude}

In the 16th century, Potosi $(3976 \mathrm{~m})$ was one of the most important cities in the world, because of its silver mountain. A Spaniard chronicle reported $100 \%$ mortality of newborns from Spaniard parents at that time, and women migrated to lower altitude cities to give birth. Several generations later, "the miracle of Saint Nicolas" occurred and a boy survived in Potosí after 6 dead siblings. Since that time, more and more children from sea level mothers (not high altitude natives) survive birth at high altitude, and the genes for postnatal adaptation to chronic hypobaric hypoxia are widespread in the 21 st century. 